\title{
THERAPEUTIC EFFECT OF TWO FLUORIDE VARNISHES INTENSIVE MODE ON WHITE SPOT LESIONS (RANDOMIZED CLINICAL TRIAL)
}

\author{
Ahmed IB Mekki ${ }^{1}{ }^{*}$ BDS, Karin ML Dowidar ${ }^{2} P h D$, Dalia AM Talaat ${ }^{3} P h D$.
}

\begin{abstract}
BACKGROUND: Fluoride varnish has been used as therapeutic agent for white spot lesions. Bio-active calcium and phosphate was included in the varnish in order to provide better subsurface remineralization. One of the most promising modifications is the addition of casein phosphopeptide amorphous calcium phosphate to fluoride varnish (CPP-ACPF).

OBJECTIVES: Evaluate the therapeutic effect of intensive application of casein phosphopeptide amorphous calcium phosphate fluoride (CPP-ACPF) varnish (MI varnish) and fluoride varnish (Duraphat) on the activity of enamel white spot lesions (WSLs) in primary teeth over a 6 weeks follow-up period.

MATERIALS AND METHODS: Forty-four participants with at least 4 active WSLs each were divided randomly into two groups. Test group (22 participants) received three times intensive professional application of MI varnish with two weeks apart. Control group (22 participants) received three times intensive professional application of Duraphat with two weeks apart. Lesions' activity in both groups was assessed using DIAGNOdent and visio-tactile lesion activity index at baseline and at 6 weeks.

RESULTS: Both groups had a significant decrease in mean DIAGNOdent scores and number of active WSLs when compared to their baseline means score. However when comparing the results of both groups, the control group (Duraphat varnish) had a significant decrease in DIAGNOdent mean scores and number of active WSLs when compared to the test group (MI varnish).

CONCLUSIONS: Intensive application of fluoride varnish decreases caries lesion activity which may lead to the arrest of the white spot lesions. Duraphat varnish is superior to MI varnish in remineralization of white spot lesions.

KEYWORDS: WSLs, remineralization, MI varnish, Duraphat.
\end{abstract}

1. Bachelor of Dentistry, Faculty of Dentistry, Alexandria University, Alexandria, Egypt

2.Professor of Pediatric Dentistry, Department of Pediatric Dentistry and Dental Public Health Department, Faculty of Dentistry,

Alexandria University, Egypt.

3.Associate Professor of Pediatric Dentistry, Dental Public Health Department, Faculty of Dentistry, Alexandria University, Egypt.

*Corresponding author:

Email: ahmedibrahimmekky@gmail.com

\section{INTRODUCTION}

Remineralization is the process by which the enamel surface is supplied with calcium and phosphate ions from external sources to increase their deposition into crystal voids in demineralized enamel. In modern dentistry, the management of white spot lesion (WSL) non-invasively through remineralization is the best way of intervention. The optimal goal of minimally invasive dental caries management is to prevent and heal the incipient lesion by inhibiting the demineralization process, preventing any further mineral loss and enhancing the remineralization process (1).

The use of a high concentration fluoride preparation on tooth surface forms a reserve of calcium fluoride, which is released when the salivary $\mathrm{pH}$ drops so it increases the rate of remineralization after caries attack. Many preventive techniques provide fluoride in high concentration to tooth surface either professionally applied as acidulated phosphate fluoride gel and sodium fluoride varnishes or home applied as fluoride rinses and fluoridated dentifrices (2).

Weinstein et al 2009(3) in their randomized equivalence trial introduced the intensive fluoride application regimen in preschooler's primary dentition by comparing 2 groups of $5 \%$ sodium fluoride varnish application regimen. The control group with standard biannual application regimen and the test group with 3 intensive applications for 6 weeks each of them were 2 weeks apart. They concluded that, although the intensive treatment was superior to the standard one, the advantage was minimal.

To produce better sub-surface remineralization, remineralizing agents should include bioavailable calcium and phosphate other than fluoride (4). Various preparations have been examined, usually containing between 1-3 mM calcium ions, with phosphate ions present at a ratio of 1:1(5) or 1.66:1 respectively (6). These preparations could be carried in a medium prepared from bovine milk, which is a good source of phosphopeptide-based products for dentistry. They include casein phosphopeptides (CPP); casein phosphopeptides with amorphous calcium phosphate (CPP-ACP); and casein phosphopeptides with amorphous calcium fluoride phosphate (CPP-ACFP) (7).

Llena et al 2015(8) in their double-blinded clinical prospective study on young permanent teeth 
proved that CPP-ACP and CPP-ACFP (MI paste \& MI paste plus) are efficient in the prevention of demineralization and increase in the remineralization rate of early enamel white spot lesions. CPP-ACFP was also superior to Duraphat varnish in the remineralization of WSLs.

One of the recently available modifications of fluoride varnish is the addition of CPP-ACP to sodium fluoride varnish (MI varnish) to provide a vehicle of bioactive calcium and phosphate ions to enhance sub-surface remineralization (9). MI varnish (CPP-ACP FV) has proven its efficacy in remineralization of WSL in in-vitro trials; however, its effectiveness is needed to be verified in clinical trials (10).

The current study aimed to compare the effectiveness of the intensive application of casein phosphopeptide amorphous calcium phosphate fluoride varnish [MI varnish (GC AMERICA INC.3737 West U.S.A.)] and 5\% (22,600 ppm) fluoride varnish [Duraphat varnish (Colgate, New York, N.Y.)] in WSLs remineralization in primary teeth. The null hypothesis was that the effect of the intensive regimen of CPP-ACPF Fluoride varnish (MI varnish) over a period of six weeks wouldn't be significantly different from that of sodium fluoride varnish (Duraphat).

\section{MATERIALS AND METHODS}

Appropriate ethical clearance was obtained from the ethics committee of Alexandria Dental University (\#IRB NO: 00010556-IORG 0008839). Study was registered in clinicaltrials.gov before recruitment started (NCT03865680). This study was a randomized clinical trial conducted on patients selected from the pediatric dentistry department clinic, Faculty of Dentistry, Alexandria University. Recruitment started at April 2019 and final data collection and statistical analysis done at March 2020. All legal guardians of participants signed an informed consent before participating in the clinical trial. Sample size was calculated using (http://powerandsamplesize.com/Calculators/Compare-2Means/2-Sample-Equality) using the following assumptions: alpha error $=5 \%$, study power $=80 \%$, mean Diagnodent reading in the two groups $=4.8$ and 3.7 , standard deviation= 2.5 (Llena et al) (8). The minimum required sample size $=81$ teeth. To make up for the potential loss to follow up after 6 months, the number was increased by $10 \%=89$ teeth $\approx 90$ per group, with a total of 180 teeth.

Inclusion criteria: 3-5 years high caries risk participants. Having at least four active white spot lesions on smooth surface of their primary anterior teeth with NYVAD's scale score 1 (11) and ICDAS score 2 (12).Participants must be medically free with no history of milk allergy,

Patients were randomly assigned using a computer generated list of random serial numbers to one of the two parallel groups (MI varnish "study group”, Duraphat fluoride varnish "control group”).The allocation was performed by a trial independent individual and the allocation ratio was intended to be equal. The allocation was done in blocks of four to ensure that both study groups had an equal number of patients (22 patients per group). Each participant included in the study was given a serial number that was used in the allocation. These numbers were written on identical sheets of paper with the group to which each child was allocated and placed inside opaque envelopes carrying the respective names of the children. A trial independent person was assigned the role of keeping the envelopes and unfolding them only at the time of the fluoride varnish application session to conceal the allocation of the participant from the examiner. Only one examiner performed the whole study procedures.

\section{Examiner reliability}

The main researcher received training for both NYVAD's scoring system and DIAGNOdent (calibration and measurements), intra examiner reliability was done using intra-class correlation coefficient (ICC) and Cohen kappa test (K) for DIAGNOdent and NYVAD's scores respectively ICC (0.92) ,K (0.81).

At the orientation visit, oral hygiene instructions were demonstrated to the legal guardian of participant, Participants received a packet of fluoridated toothpaste (500 ppm of fluoride) and a toothbrush. The caregiver was instructed to put only pea sized toothpaste while brushing the participant's teeth (13) and to avoid giving the participant any other fluoride supplements or professionally applied ones during the study period.

At the baseline visit, patient's teeth were cleaned using prophylaxis brush without a paste. DIAGNOdent (Kavo Dental Corporation Bismarck ring 39, Biberach, 88400, Germany) was calibrated against the device's own disk. Under cotton roll isolation a baseline zero value for each patient was obtained from caries-free buccal surface of an anterior tooth. The selected tooth was written on the patient chart as a reference as it was used for further measurements. The reference teeth were not included in the statistical analysis of the study. DIAGNOdent scores ranges from (0-99) the greater the score the greater the demineralization in the caries lesion. Each WSL had three readings recorded and the three scores had been averaged to get the final score. The score of each lesion was registered in the patient's chart. At the end of the visit, each participant received fluoride varnish application under partial isolation. Both fluoride varnishes were applied according to their manufacturer's instructions (14, 15). The participants were instructed not to eat for the next four hours (14).

At two and four weeks follow up visits, the patient's teeth were cleaned using a prophylaxis brush with no paste and the fluoride varnishes were re-applied as previously mentioned. Oral hygiene instructions were given to both patient and the caregiver.

At the six week visit, the patient's teeth were cleaned using prophylaxis brush without a paste, lesion activity score of each lesion was recorded in the patient's chart following the Nyvad's (11) scoring system.DIAGNOdent scores of the participant's selected teeth were registered in his chart as mentioned before. DIAGNOdent scores were correlated to the white spot lesion activity using Pinelli et al (16) Cut-off points that included the following scores:

0-4 arrested or inactive lesion $>4$ active lesion. 


\section{Study outcome}

Change in white spot lesion mineralization status was assessed after intensive application of either MI varnish or Duraphat varnish using:

The change in DIAGNOdent scores either indicates deminrealization when scores increased or remineralization when scores decrease.

Change in white spot lesion activity state after intensive application of either MI varnish or Duraphat varnish using:

The change in NYVAD'S criteria scores. Either receiving score 1 which indicates active lesion or score 4 which means as inactive caries lesion.

DIAGNOdent cut-off points.

Statistical analysis

Differences between groups in DIAGNOdent readings was assessed using independet $t$ test while within group differences was assesed using paired t test. Fisher's Exact test and Chi Square tests were applied to lesion activity comparsions and McNemar for paired nominal data. Percent redcution was calculated as [(Values after intervention- vales before)/Before]X100.Significance level was set at 0.05. Data was analyzed using IBM SPSS statistical software (version 25).

\section{RESULTS}

Thestudy sample included 44 children (25 males, 19 females) with mean age $3.8 \pm 0.70$ having 180 active WSLs (Fig 1). There was no significant difference between the two groups in terms of sex ratio $(p=0.76)$ or mean age $(p=0.85)$.

The assessment of DIAGNOdent scores at baseline revealed no significant difference between the MI varnish with a mean score of $8.56( \pm 2.40)$ and Duraphat varnish with mean score $7.6( \pm 3.27) p=0.12$. At the end of 6 weeks follow up period (intensive fluoride varnish application period) the results showed a significant decrease in the DIAGNOdent scores of the test group (MI varnish) with mean score $4.56( \pm 1.54)$ with a $\mathrm{p}<0.0001$. The test group (MI varnish) showed a percentage of reduction 46.99 ( \pm 9.11$)$. The control group (Duraphat varnish) showed a significant decrease in their mean DIAGNOdent scores 3.46( \pm 2.17$)$ with a $\mathrm{p}<0.0001$. The control group (Duraphat varnish) showed percentage of reduction $56.55( \pm 12.62)$.

Comparing the decrease in mean DIAGNOdent score between both groups the control group showed a significant difference from the test one (MI-varnish) with a $(p<0.001)$. By comparing the percent of reduction between both groups the control group (Duraphat) showed significant difference from the test group (MI) with a $\mathrm{p}<0.0001$ (Table 1 ).

The assessment of Visio-tactile lesion activity score according to Nyvad's criteria (14) at baseline revealed no significant difference between both groups as $90(100 \%)$ active lesions were found in both groups. At the $6^{\text {th }}$-week measurement test group (MI varnish) had a significant reduction in the number of active white spot lesions reaching 60(66.7\%) active lesions $\mathrm{p}<0.0001$, whereas control group (Duraphat varnish) had a significant reduction in active white spot lesions reaching $35(40 \%)$ active lesions $\mathrm{p}<0.0001$. When comparing the results of both groups, the control group (Duraphat) showed a significant difference from the test group (MI varnish) $\mathrm{p}=0.002$ (Table 2).

By using DIAGNOdent cut-off points for lesion activity detection (18), at baseline $96.7 \%$ of the test group (MI varnish) were active lesions and $88.9 \%$ of the control group (Duraphat) were active lesions with no significant difference between both groups. At 6 weeks, the test group (MI varnish) had a significant reduction in the number of active lesions reaching $61.1 \% \quad \mathrm{p}<0.002$ whereas the control group (Duraphat) had significant reduction in active lesion reaching $27.8 \% \quad \mathrm{p}<0.002$. Comparing the reduction in the number of active lesions between both groups, the control group (Duraphat varnish) showed a significant difference in the decrease of number of active WSLs from test group (MI varnish) $\mathrm{p}<0.0001$ (Table3)

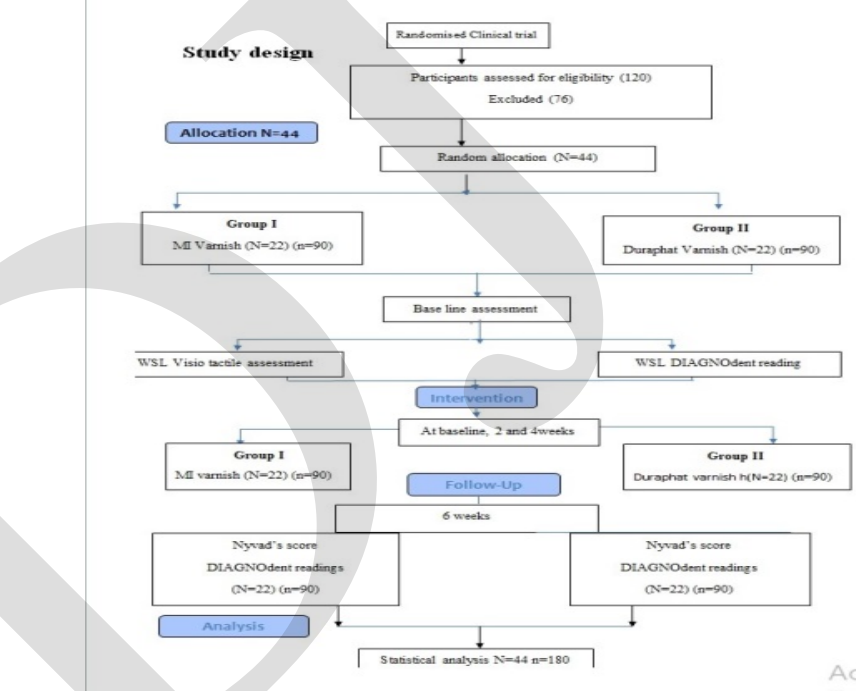

Figure 1: Consort flow chart

* $\mathrm{N}$ : number of participants , $\mathrm{n}$ : number of lesions

Table 1: Comparison of DIAGNOdent readings between the test and control groups at baseline and after 6 weeks

\begin{tabular}{|l|l|l|l||}
\hline \multirow{3}{*}{$\begin{array}{l}\text { Time } \\
\text { points }\end{array}$} & $\begin{array}{l}\text { MI varnish } \\
(\mathrm{N}=22, \\
\mathrm{n}=90)\end{array}$ & $\begin{array}{l}\text { Duraphat } \\
\text { varnish } \\
(\mathrm{N}=22, \\
\mathrm{n}=90)\end{array}$ & \multirow{2}{*||}{ P value } \\
\cline { 2 - 4 } & Mean (SD) & \\
\hline Baseline & $8.56(2.40)$ & $7.60(3.27)$ & 0.12 \\
\hline 6 weeks & $4.56(1.54)$ & $3.46(2.17)$ & $<0.0001^{*}$ \\
\hline$P$ value & $<0.0001^{*}$ & $<0.0001^{*}$ & \\
\hline $\begin{array}{l}\text { \% } \\
\text { reduction }\end{array}$ & $\begin{array}{l}46.99 \\
(9.11)\end{array}$ & $56.55(12.62)$ & $<0.0001^{*}$ \\
\hline
\end{tabular}

*Statistically significant at $p$ value $\leq 0.05$. Differences between groups in DIAGNOdent readings was assessed using independent $t$ test while within group differences was assessed using paired t test 
Table 2: Comparison of lesion activity score between the test and control groups at baseline and after 6 weeks

\begin{tabular}{|c|c|c|c|c|}
\hline \multirow{2}{*}{\multicolumn{2}{|c|}{ Time points }} & $\begin{array}{l}\text { MI varnish } \\
(\mathrm{N}=22,\end{array}$ & $\begin{array}{l}\text { Duraphat } \\
\text { varnish }\end{array}$ & \multirow[t]{2}{*}{$P$ value } \\
\hline & & \multicolumn{2}{|l|}{ n (\%) } & \\
\hline \multirow[t]{2}{*}{ Baseline } & Active & $90(100 \%)$ & $90(100 \%)$ & \multirow[t]{2}{*}{1} \\
\hline & Inactive & 0 & 0 & \\
\hline \multirow[t]{2}{*}{6 weeks } & Active & 60 (61.1\%) & $35(27.8 \%)$ & \multirow[t]{2}{*}{$<0.002 *$} \\
\hline & Inactive & $30(38.9 \%)$ & $55(72.2 \%)$ & \\
\hline \multicolumn{2}{|l|}{$P$ value } & $<0.0001^{*}$ & $<0.0001^{*}$ & \\
\hline
\end{tabular}

*Statistically significant at $p$ value $\leq 0.05$. Fisher's Exact test and Chi Square tests were applied to lesion activity comparsions and McNemar for paired nominal data

Table 3: Comparison of DIAGNOdent cut-off points between the test and control groups at baseline and after 6 weeks

\begin{tabular}{|c|c|c|c|c|}
\hline \multicolumn{2}{|c|}{ Time points } & $\begin{array}{l}\text { MI } \\
\text { varnish }\end{array}$ & $\begin{array}{l}\text { Durapha } \\
\text { t varnish }\end{array}$ & \multirow[t]{2}{*}{$P$ value } \\
\hline & & \multicolumn{2}{|l|}{ n (\%) } & \\
\hline \multirow[t]{2}{*}{$\begin{array}{l}\text { Baselin } \\
\text { e }\end{array}$} & Active & $\begin{array}{l}87 \\
(96.7 \%\end{array}$ & $\begin{array}{l}80 \\
(88.9 \%)\end{array}$ & \multirow[t]{2}{*}{0.06} \\
\hline & $\begin{array}{l}\text { Inactiv } \\
\mathrm{e}\end{array}$ & $\begin{array}{l}3 \\
(3.3 \%)\end{array}$ & $\begin{array}{l}10 \\
(11.1 \%)\end{array}$ & \\
\hline \multirow[t]{2}{*}{6 weeks } & Active & $\begin{array}{l}55 \\
(61.1 \%\end{array}$ & $\begin{array}{l}25 \\
(27.8 \%)\end{array}$ & \multirow[t]{2}{*}{$\begin{array}{l}<0.0001 \\
*\end{array}$} \\
\hline & $\begin{array}{l}\text { Inactiv } \\
\text { e }\end{array}$ & $\begin{array}{l}35 \\
(38.9 \% \\
)\end{array}$ & $\begin{array}{l}65 \\
(72.2 \%)\end{array}$ & \\
\hline \multicolumn{2}{|l|}{$P$ value } & $0.03 *$ & $0.04^{*}$ & \\
\hline
\end{tabular}

*Statistically significant at $p$ value $\leq 0.05$. Fisher's Exact test and Chi Square tests were applied to lesion activity comparsions and McNemar for paired nominal data

\section{DISCUSSION}

Fluoride varnish is widely accepted by the patient especially at a young age due to ease of application and acceptable taste (17). Semi-annual or quarterly application is the gold standard for the remineralization of white spot lesions (18-19). Casein phosphopeptide amorphous calcium phosphate-based fluoride varnish (MI varnish) is proven effective in WSL remineralization in in-vitro trials $(20,21)$, as it provides a vehicle of bioactive calcium and phosphate ions to enhance sub-surface remineralization.

Monitoring white spot lesions progression in a non-invasive way can be achieved using either Visio tactile assessments (11) or laser fluorescence devices such as DIAGNOdent, which serves as a promising device to observe the change in mineral content and the lesion depth of white spot lesions (22-24).
To our knowledge, there are no published clinical trials comparing CPP-ACPF Fluoride Varnish (MI varnish) and sodium fluoride varnish (Duraphat). The results of this study showed that the test group (MI varnish) at the end of the six weeks (intensive fluoride application period) had a significant decrease in their DIAGNOdent scores when compared to their baseline scores.as CPP-ACP stabilizes bio-active calcium and phosphate ions in both supra-gingival plaque and the deeper layers of WSLs which leads to better remineralization and physical properties of the WSLs. This is in agreement with Savas et al (25) who evaluated the remineralization capacity of MI varnish on white spot lesions using DIAGNOdent scores. Moreover, the control group (Duraphat) had a significant decrease in their WSLs DIAGNOdent scores as proven in several in vivo trials $(26,27)$.

The number of active white spot lesions at the end of the six weeks intensive fluoride varnish application period decreased significantly in both test (MI varnish) and control groups (Duraphat). As there is solid proof that $\mathrm{NaF}$ varnishes are indeed efficient in increasing the absorption of fluoride and reducing the enamel's mineral loss under various conditions. Also, NAF varnish intensive application has been proven effective in remineralization of WSLs this was shown in Ferreira JM et al (28) randomized clinical trial who used four consecutive sodium fluoride varnish intensive application as a treatment regimen for WSLs.

DIAGNOdent cut-off points that were developed by Pinelli et al (16) to detect WSL activity using DIAGNOdent scores in vivo, it has been used in this study to correlate between DIAGNOdent readings and the results from Visio-tactile assessment of WSL (11). These results showed a significant decrease in the number of active lesions in both groups when compared to their baseline data, which is in agreement with the previous results of the Visio-tactile assessment.

Comparing the results of both groups, the null hypothesis is rejected as at the end of the intensive application period control group (Duraphat) had significantly better results than the test group (MI varnish) in both reductions of laser fluorescence scores and number of active lesions. This finding is in agreement with Mohd Said et al (29) who stated that fluoride varnish (Duraphat) is superior to CPP-ACPF varnish (MI varnish) in remineralization of enamel caries lesions. This could be clarified based on the high release of phosphate ions from MI varnish (30), as high levels of inorganic phosphate may be unfavorable for the formation of loosely bound fluoride reservoirs as they can reduce the formation of bioactive calcium fluoride $(\mathrm{CaF}+)$ and calcium fluoride (CaF2) and favors the formation of Fluor-apatite. The formation of ectopic Fluor-apatite will dramatically decrease the activity and availability of fluoride ions (31). On the other hand,Tuloglu et al(24) in their in vitro study stated that CPP-ACPF Fluoride varnish (MI varnish) is superior to Duraphat in caries lesions remineralization due to the anti-cariogenic mechanism of CPP-ACP, which may involve the incorporation of Nano-complexes into plaque and onto the tooth surface. These localized CPP-ACP Nano 
complexes have been purported to buffer the activity of free calcium and phosphate ions, thereby maintaining a state of super saturation with tooth enamel, preventing enamel demineralization and promoting remineralization. The intensive application of MI varnish in the current study might have increased the bio-available phosphate ion concentration dramatically in saliva so yields to less formation of Calcium fluoride (reservoirs) on the tooth surface and formation of more ectopic Fluor-apatite which leads to decreased amount of free fluoride needed for remineralization. (29) On the other hand, intensive application of Duraphat fluoride varnish allowed the formation of more Calcium fluoride (reservoirs) on tooth surfaces leading to better resistance to further acid attacks by providing more free fluoride ions to incorporate in WSLs remineralization.The main limitation of this study was Participants'compliance for oral hygiene instructions as it varies from one participant to another.

\section{CONCLUSIONS}

The Intensive mode of fluoride varnish application decreased caries lesion activity significantly, leading to the arrest of the white spot lesions.

Duraphat varnish was superior to MI varnish in remineralization of white spot lesions when applied in an intensive mode.

\section{CONFLICT OF INTEREST}

The authors declare that they have no conflicts of interest.

\section{REFERENCES}

1. Cochrane NJ, Cai F, Huq NL, Burrow MF, Reynolds EC. New Approaches to enhanced remineralization of tooth enamel. J Dent Res. 2010; 1187-97.

2. American Academy of Pediatric Dentistry.Guideline on fluoride therapy.Ped Dent. 2014;E176-8.

3. Weinstein P, Spiekerman C, Milgrom P. Randomized equivalence trial of intensive and semiannual applications of fluoride varnish in the primary dentition. Caries research. 2009;484-90.

4. RehderNeto FC, Maeda FA, Turssi CP, Serra MC. Potential agents to control enamel caries-like lesions. J Dent 2009; 786-90.

5. Wefel JS, Harless JD. The use of saturated DCPD in remineralization of artificial caries lesions in vitro. J Dent Res. 1987 ;1640-3.

6. Iijima Y, Takagi O, Ruben J, Arends J. In vitro remineralization of in vivo and in vitro formed enamel lesions.Caries research. 1999:206-13.

7. Aimutis WR. Bioactive properties of milk proteins focus on anticariogenesis. J Nutr. 2004:989-95.

8. Llena C, Leyda AM, Forner L. CPP-ACP and CPPACFP versus fluoride varnish in remineralisation of early caries lesions. A prospective study.Eur J Paediatr Dent. 2015:181-6.

9. Thakkar PJ, Badakar CM, Hugar SM, Hallikerimath S, Patel PM, Shah P. An in vitro comparison of casein phosphopeptide-amorphous calcium phosphate paste, casein phosphopeptide-amorphous calcium phosphate paste with fluoride and casein phosphopeptideamorphous calcium phosphate varnish on the inhibition of demineralization and promotion of remineralization of enamel.Journal of Indian Society of Pedodontics and Preventive Dentistry. 2017 Oct $1: 312$.

10. Salman $\mathrm{nr}$, el-tekeya $\mathrm{mm}$, bakry $\mathrm{ns,} \mathrm{soliman} \mathrm{s.}$ remineralization effect of fluoride varnish containing casein phosphopeptide amorphous calcium phosphate on caries-like lesions in primary teeth (in vitro study). alexandriadental journal. 2019 apr 1;13-6.

11. Nyvad B, Baelum V. Nyvad criteria for caries lesion activity and severity assessment: a validated approach for clinical management and research. Caries research. 2018:397-405.

12. Dikmen B. Icdas II criteria (international caries detection and assessment system).Journal of Istanbul University Faculty of Dentistry.2015:63.

13. Wright JT, Hanson N, Ristic H, Whall CW, Estrich CG, Zentz RR. Fluoride toothpaste efficacy and safety in children younger than 6 years: a systematic review. The Journal of the American Dental Association.2014 Feb 1:182-9.

14. Colgate.DuraphatVarnish.https://www.colgateprofessi onal.com.au/products/products-list/colgate-durapathvarnish-single-dose (accessed 20/1/2020).

15. GC .MI varnish.https://www.gceurope.com/products/mivarnis h/ (accessed 20/1/2020).

16. Pinelli C, Serra MC, Loffredo LD. Validity and reproducibility of a laser fluorescence system for detecting the activity of white-spot lesions on free smooth surfaces in vivo. Caries research. 2002;19-24.

17. Autio-Gold JT, Courts F. Assessing the effect of fluoride varnish on early enamel carious lesions in the primary dentition. J Am Dent Assoc 2001:1247-1253.

18. American Academy of Pediatric Dentistry.Guideline on Fluoride Therapy, 2018 http://www. aapd.org/media/Policies_Guidelines.

P_FluorideUse.pdf.

19. Restrepo M, Bussaneli DG, Jeremias F, Cordeiro RC, Raveli DB, MagalhãesAC et al. Control of white spot lesions with use of fluoride varnish or chlorhexidine gel during orthodontic treatment a randomized clinical trial. Journal of Clinical Pediatric Dentistry. 2016 Oct:274-80.

20. Pithon MM, dos Santos MJ, Andrade CS, LeãoFilho JC, Braz AK, de Araujo RE et al. Effectiveness of varnish with CPP-ACP in prevention of caries lesions around orthodontic brackets: an OCT evaluation. European journal of orthodontics.2014 Jul 4:177-82.

21. .Tuloglu N, Bayrak S, Tunc ES, Ozer F. Effect of fluoride varnish with added casein phosphopeptideamorphous calcium phosphate on the acid resistance of the primary enamel. BMC oral health. 2016 Dec;103.

22. Andersson A, Sköld-Larsson K, Haligren A, Petersson LG, Twetman S, Hallgren A. Effect of a dental cream containing amorphous cream phosphate complexes on white spot lesion regression assessed by laser fluorescence. Oral health \& preventive dentistry. 2007 Jun 1;5.

23. Shi XQ, Tranæus S, Angmar-Månsson B. Validation of DIAGNOdent for quantification of smooth-surface 
caries: an in vitro study. ActaOdontologicaScandinavica.2001 Jan 1:74-8.

24. Mendes FM, Siqueira WL, Mazzitelli JF, Pinheiro SL, Bengtson AL. Performance of DIAGNOdent for detection and quantification of smooth-surface caries in primary teeth. Journal of dentistry. 2005 Jan :79-84.

25. Savas S, Kavrik F, Kucukyilmaz E. Evaluation of the remineralization capacity of CPP-ACP containing fluoride varnish by different quantitative methods. Journal of Applied Oral Science. 2016 Jun:198-203.

26. Du M, Cheng N, Tai B, Jiang H, Li J, Bian Z. Randomized controlled trial on fluoride varnish application for treatment of white spot lesion after fixed orthodontic treatment. Clinical oral investigations.2012 Apr 1:463-8.

27. Perrini F, Lombardo L, Arreghini A, Medori S, Siciliani G. Caries prevention during orthodontic treatment: In-vivo assessment of high-fluoride varnish to prevent white spot lesions. American Journal of Orthodontics and Dentofacial Orthopedics. 2016 Feb $1 ; 238-43$.
28. Ferreira JM, Aragão AK, Rosa AD, Sampaio FC, Menezes VA. Therapeutic effect of two Sfluoride varnishes on white spot lesions: a randomized clinical trial. Brazilian oral research. 2009 Dec:446-51.

29. Mohd Said SN, Ekambaram M, Yiu CK. Effect of different fluoride varnishes on remineralization of artificial enamel carious lesions. International journal of paediatric dentistry. 2017 May:163-73.

30. .Cochrane NJ, Shen P, Yuan Y, Reynolds EC. Ion release from calcium and fluoride containing dental varnishes.Aust Dent J 2014; 59: 100-105.

31. Christoffersen J, Christoffersen MR, Kibalczyc W, Perdok WG. Kinetics of dissolution and growth of calcium fluoride and effects of phosphate.ActaOdontologicaScandinavica. 1988Jan ;325-36.

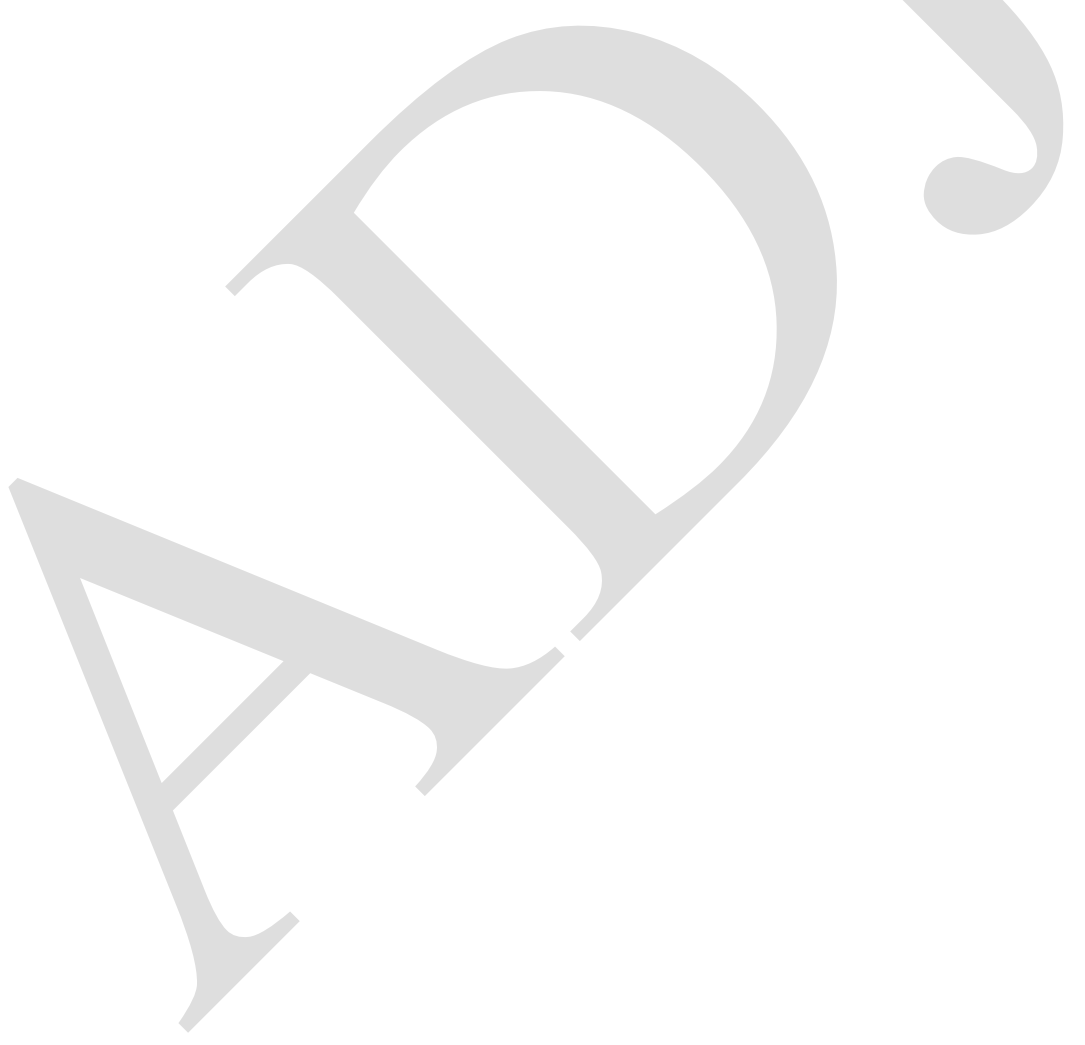

